\title{
Fitting simulation evaluation on personalized avatars
}

\begin{abstract}
This study examines visualization of fit of select garment designs on two types of virtual avatars: a personalized avatar developed through self-body scanning technology, and a selected avatar from a pre-programmed software program. The purpose is to increase understanding of the visual elements, accuracy and reliability of fitting simulations. For this exploratory comparative analysis, a purposive sample of design students designed five garments on a custom avatar using the Kinect system and an avatar selected from the Optitex computer program that most closely resembled the designer. Design professionals rated the appearance, smoothness and fit of the front, back and side views of each garment and avatar, and included qualitative comments. Results show a low range of difference but the pre-programmed avatar scored higher on appearance, smoothness and fit. Qualitative responses suggest the pre-programmed avatar is more aesthetically pleasing but the self scanned avatar showed more accurate body proportions of the torso and drape of fabric relative to the body. Implications for further research are included.
\end{abstract}

Keywords: fitting simulation, avatar, 3D body scanning
Volume 4 Issue 2 - 2018

\author{
Shu Hwa Lin,' Rayneld R Johnson, ${ }^{2}$ Ju-Young \\ Kangl \\ 'University of Hawaii at Manoa, USA \\ ${ }^{2}$ Wayne State University, USA
}

Correspondence: Shu Hwa Lin, Associate Professor, Department of Family \& Consumer Sciences, University of Hawaii at Manoa, Honolulu, HI 96822, USA, Tel 808-956-2245, Email shulin@hawaii.edu

Received: January 08, 2018 | Published: March 02, 2018

\section{Introduction}

Body scanning systems and computerized avatars have been developing to offer consumers new options in apparel fit and virtual try-on. ${ }^{1,2}$ Consumers can create a personalized avatar by first selecting a programmed avatar whose measurements are closest to one's own and choosing like personal features such as hair, skin color and eyes. Personalized avatars are being used in a variety of applications such as virtual try-on and the design process to facilitate visualization of garment fit.

Selected retailers and apparel companies offer consumers an opportunity to personalize a programmed avatar to try-on and visualize a garment prior to purchase. ${ }^{3}$ Fit is cited as a major consumer complaint and virtual try-on is intended to increase consumer satisfaction and reduce customer returns., ${ }^{3,4}$ More recent technical advancements such as the virtual try-on, gives additional physical simulation and an animated virtual avatar in an attempt to display garment movement and fabric drape on the body. ${ }^{3-6}$ Another method to provide more realism and mirror imaging in virtual avatar representation is through 3D body scanning systems such as Kinect. Kinect, developed by Microsoft, is an affordable home device that senses motion as output. ${ }^{7}$ Kinect employs a revolutionary technique designed for games, entertainment and fitness (Wii-Fit). ${ }^{8,9}$

For the design process, personalized programmed avatars also assist a designer's visualization of a garment before production. ${ }^{10}$ However, if the designer is creating a garment for himself or herself, an avatar that matches one's own body as closely as possible offers a more realistic visualization of the garment. Computer simulation is providing the most effective way to detect fitting issues and construction problems that may occur during the production process. Also, the selection of appropriate avatars for mass production can be challenging for manufacturers. This study explores and compares fitting simulation issues related to two types of avatars.

\section{Research questions}

a. RQ1: Does using a 3D body scanning system such as Kinect to scan oneself and create an avatar versus using a customized programmed avatar in the design process influence a better visualization of fit?

b. RQ2: Do different virtual avatars have different fitting results?

This exploratory study investigates the differences in fitting simulation on personalized avatar and customized programmed avatars.

\section{Method}

A purposive convenience sample of 20 fashion undergraduate design students including 19 females and one male voluntarily participated in this exploratory study and created two avatars using two different methods. The design students were familiar with the 3D Body Scanning Kinect technology. The participants were recruited from an existing large sample of 299 students who had experience with 3D body scanning. The participants also had knowledge of the Optitex computer software. Additionally, using selected criteria of appearance, smoothness and fit, three design professionals evaluated the front, side, and back views of each avatar and included qualitative comments based on their observations.

\section{Procedure}

For the fitting simulation, participants were directed to create two virtual avatars using two different methods. For one method, using the Kinect system, participants had their bodies scanned and a custom avatar was generated. For the other method, participants used a programmed avatar from 3D CAD virtual prototype software (i.e., Optitex PDS program). Participants designed and created 3D patterns for basic garments such as skirts, t-shirts, tank tops, pants, 
and sleeveless dresses. Then, they inserted their desired fabric print images into 3D patterns and finalized creation of the 3D virtual garments using the Optitex PDS program.

To create fitting simulations on self-body scanned avatars (i.e., personalized avatar), a ply file was converted to obj file using Mesh Lab software because obj files are an appropriate format for importing 3D body scan data into the Optitex PDS program. The obj was checked in the "plugins list" of the 3D menu in the Optitex PDS program in order to import the 3D geometry of the obj file format. When the personalized avatar was loaded, the obj file was selected, and then the self-body scanned avatar was shown in the 3D model window. The personalized avatar by $90^{\circ}$ was rotated to see the correct figure. Participants placed their 3D patterns/3D virtual garment pieces on the personalized avatar by clicking the "place cloth" button in the 3D toolbar. Using the "3D move or rotate piece tool" in the 3D toolbar, all $3 \mathrm{D}$ virtual garment pieces were well positioned in order to create the 3D virtual garment. Lastly, by clicking the "simulate draping" button, a 3D draping simulation was shown on the personalized avatar.

To create fitting simulations on personalized avatars (Avatar 1), an avatar from the Optitex PDS program (Avatar 2) was selected based on measurements closest to oneself. Participants placed their 3D patterns/3D virtual garment pieces on the customized programmed avatar. A 3D draping simulation was performed by adjusting the positions of the 3D patterns/3D virtual garment pieces on the personalized avatar and clicking the "simulate draping" button. Thus, the participants draped and fitted the $3 \mathrm{D}$ virtual garments on customized programmed avatars (Table 1).

Table I Generating avatars by 3D body scans and software program

\begin{tabular}{lll}
\hline & Avatar 1 & Avatar 2 \\
\hline \multirow{3}{*}{ Method } & $\begin{array}{l}\text { Using 3D body scanning } \\
\text { Kinect system to create } \\
\text { personalized avatar }\end{array}$ & $\begin{array}{l}\text { Customized programmed } \\
\text { figures }\end{array}$ \\
$\begin{array}{l}\text { Try-on } \\
\text { Simulation }\end{array}$ & $\begin{array}{l}\text { Using Optitex program to } \\
\text { try-on clothing and display } \\
\text { computer images }\end{array}$ & $\begin{array}{l}\text { Using Optitex program } \\
\text { to try-on clothing and } \\
\text { display computer images }\end{array}$ \\
\end{tabular}

To compare fitting simulations of personalized avatars (Avatar 1) and customized programmed avatars (Avatar 2), screenshots of the final fitting simulation images of each self-body scanned avatar (Avatar 1) and customized programmed avatar (Avatar 2) were made. Three design professionals who would compare fitting simulations were selected (Figure 1). Students were able to simulate fitting without constructing garments as well as reduce time by using CAD technology. All evaluations were conducted by three design professionals.

\section{Instrument}

A closed rating scale of 1-10, 1 being "Unsatisfactory" and 10 being "Satisfactory" was devised to evaluate the Front, Back and Side views on Appearance, Smoothness and Fit of each of the five garments designs. Following the development of avatars and garment designs, independently and objectively, using the rating scale, three design professionals rated the Front, Side and Back views of each avatar on Appearance, Smoothness, and Fit. Numerical ratings were recorded in a table for each garment design. The mean scores of the ratings were also calculated.

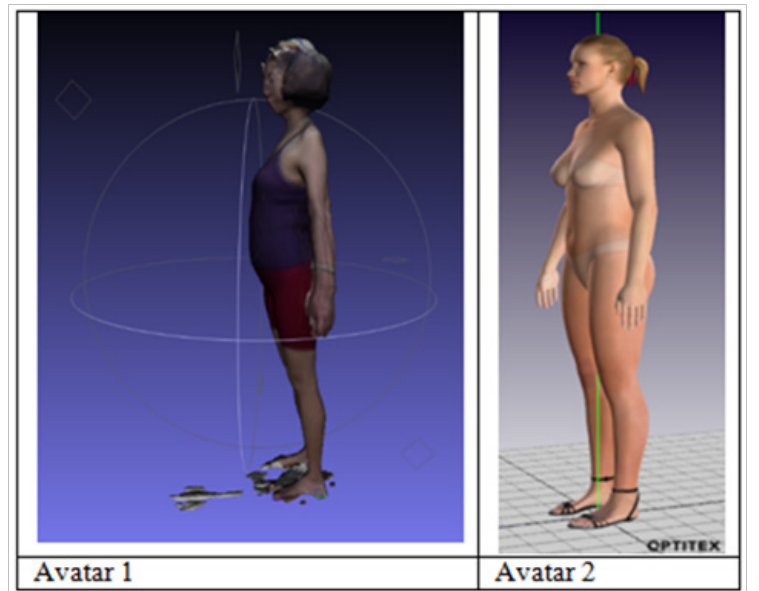

Figure I Two types of avatars:Avatar I = personalized avatar (left) and Avatar2 $=$ program avatar (right).

\section{Results}

A representative example of images depicting fitting simulations on the two types of avatars is presented, and the evaluators' ratings and mean scores of the select criteria for the basic designs: A-line Skirt, T-shirt, Top and Pants, Woman's Dress, and Sleeveless Straight Dress are listed in the following tables. In five tables present the evaluation of fitting simulation and five pairs of figures compare the different images of fitting simulation of two types of avatars.

\section{Evaluation of A-line skirt}

Table 2 displays the ratings for the A-line Skirt with plaid fabric. Plaid was used to help detect any fitting issues, such as an uneven weave or hem. Higher ratings for appearance, smoothness and fit were assessed for Avatar 2. However, Avatar 2 presents a more aesthetic image. Also there is an apparent difference on the side view of the A-line skirt simulation. The image of Avatar 1 shows less fabric plaid and Avatar 2 shows a more even fabric plaid weave on the side view (Figure 2). Overall, the front view of Avatar 1 presents a rounded and curved appearance, while Avatar 2 shows a flatter appearance in the front view.

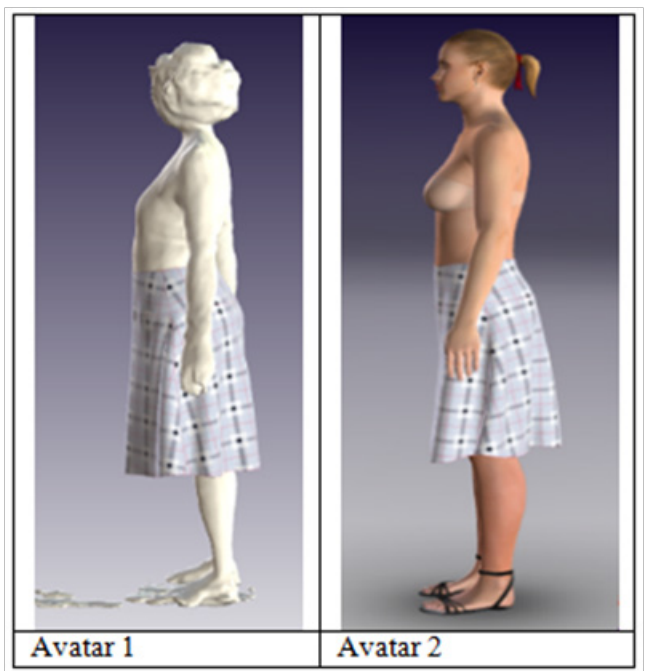

Figure 2 A-line skirt simulation on Avatar I (left) and Avatar 2 (right). 
Table 2 Evaluation of A-line Skirt

\begin{tabular}{|c|c|c|c|c|c|c|c|c|c|c|}
\hline & \multicolumn{4}{|c|}{ Avatar 1} & \multirow{2}{*}{$\begin{array}{l}\text { Mean } \\
8.3\end{array}$} & \multicolumn{4}{|c|}{ Avatar 2} & \multirow{2}{*}{$\begin{array}{l}\text { Mean } \\
10\end{array}$} \\
\hline & $\mathrm{F}$ & 8 & 8 & 8 & & $\mathrm{~F}$ & 10 & 10 & 10 & \\
\hline \multirow[t]{3}{*}{ Appearance } & $\mathrm{S}$ & 9 & 8 & 8 & 9.6 & S & 9 & 10 & 10 & 9.6 \\
\hline & B & 10 & 10 & 10 & 8.3 & B & 10 & 10 & 10 & 10 \\
\hline & $\mathrm{F}$ & 8 & 8 & 9 & 8.3 & 10 & 10 & 10 & 10 & 10 \\
\hline \multirow[t]{3}{*}{ Smoothness } & $\mathrm{S}$ & 8 & 7 & 9 & 8 & 10 & 10 & 10 & 10 & 10 \\
\hline & B & 8 & 8 & 9 & 8.3 & 10 & 10 & 10 & 10 & 10 \\
\hline & $\mathrm{F}$ & 9 & 9 & 9 & 9 & $\mathrm{~F}$ & 9 & 9 & 10 & 9.3 \\
\hline \multirow[t]{2}{*}{ Fit } & $\mathrm{S}$ & 9 & 9 & 9 & 9 & $\mathrm{~S}$ & 9 & 10 & 10 & 9.6 \\
\hline & B & 9 & 9 & 9 & 9 & B & 10 & 10 & 10 & 10 \\
\hline
\end{tabular}

Note: F, Front view; S, Side view; B, Back view

\section{Evaluation of T-shirt}

Evaluations for the T-shirt are presented in Table 3. Higher ratings in appearance, smoothness and fit were assessed for Avatar 2. However, it was noted that the smooth wrinkle free appearance of Avatar 2 did not accurately reflect the drape of the fabric, whereas Avatar 1 did reflect more realistic creases in the fabric. Due to experimental apparel design, fitting issues - appear less smooth on Avatar 1 than Avatar 2. This less smooth appearance suggests the need for pattern adjustment (Figure 3).

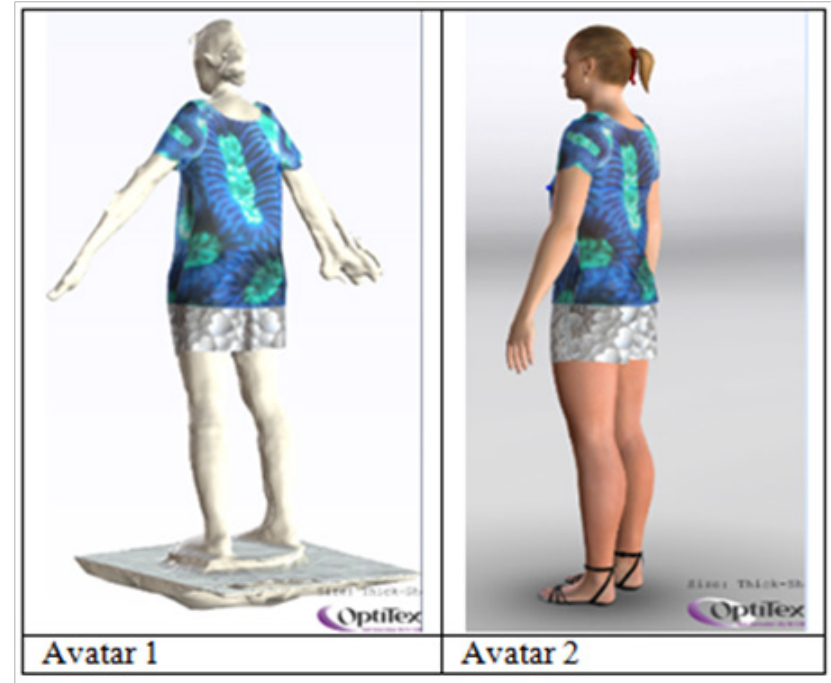

Figure 3 T-shirt simulation on Avatar I (left) and Avatar 2 (right)

\section{Evaluation of tank top and pants}

The evaluation of the Tank Top and Pants are presented in Table 4. The ratings are very close, but the scores for the Avatar 2 are slightly higher. Differences were viewed mainly in the torso and at the hem of the garments. The Avatar 1 portrayed the drape of the lightweight fabric and relativity of the fabric to the body more accurately. Due to experimental apparel design, fitting issues may be less smooth on Avatar 1 than Avatar 2, such as lower torso. However, the lower torso fit was acceptable except the hem area (Figure 4).

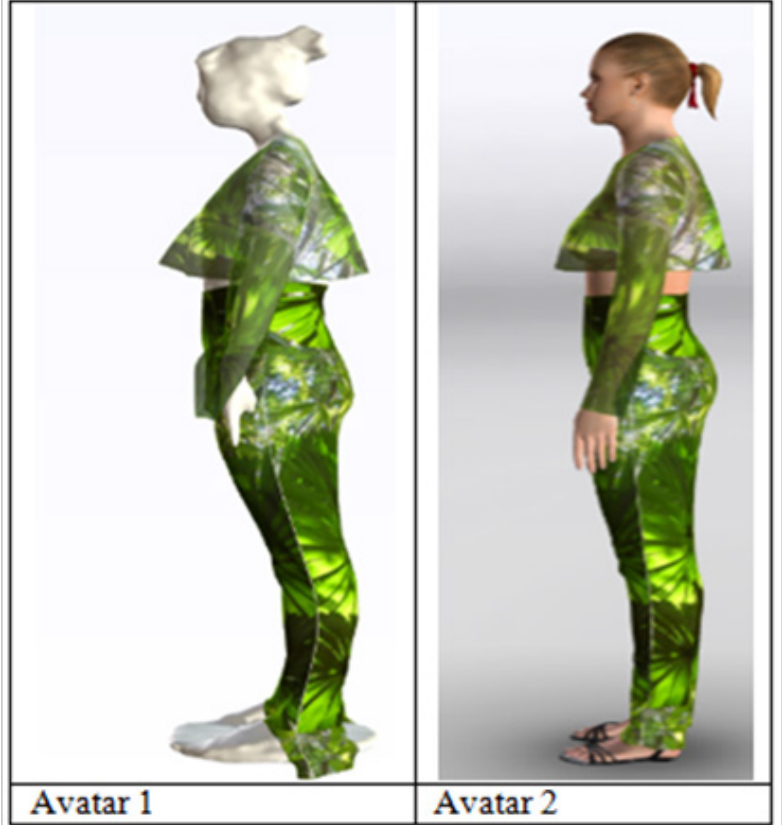

Figure 4 Top and Pants simulation on Avatar I (left) and Avatar 2 (right)

\section{Evaluation of women's dress}

Table 5 displays the ratings for the Woman's Dress with bright colored pattern and fabric with straight, parallel vertical lines. The vertical straight, parallel lines assist in detecting the fitting problems by making the smoothness and wrinkles more apparent. Slightly higher scores are indicated for Avatar 2 versus Avatar 1. The fitting simulation displayed good appearance, smoothness and fit in the bodice area. Differences were judged in the torso and hem. More natural wrinkles were evident in Avatar 1. Additionally, the appearance of the wrinkle at the hemline on Avatar 1 suggests potential pattern alterations (Figure 5).

\section{Evaluation of sleeveless straight dress}

This straight one-piece dress presents a fashionable contemporary look. The results of the evaluation of the contemporary Sleeveless 
Straight Dress simulation on both avatars are presented in Table 6. Higher ratings are indicated for Avatar 2 compared to Avatar 1. Also, fit was evident in the bodice of both avatars. But differences are apparent in the drape of the hem. The skirt and back torso look very fitted and smooth on Avatar 2. On the other hand, more natural wrinkles appear on Avatar 1. Specifically, the appearance of a wrinkle at the hem on the front suggests additional room for the chest is needed in the pattern to fit Avatar 1 (Figure 6).

Table 3 Evaluation of T-shirt

\begin{tabular}{|c|c|c|c|c|c|c|c|c|c|c|}
\hline & \multicolumn{4}{|c|}{ Avatar 1} & \multirow{2}{*}{$\begin{array}{l}\text { Mean } \\
6.6\end{array}$} & \multicolumn{4}{|c|}{ Avatar 2} & \multirow{2}{*}{$\begin{array}{l}\text { Mean } \\
8.3\end{array}$} \\
\hline & $\mathrm{F}$ & 6 & 6 & 8 & & $\mathrm{~F}$ & 8 & 8 & 9 & \\
\hline \multirow[t]{3}{*}{ Appearance } & $\mathrm{S}$ & 6 & 6 & 8 & 6.6 & $\mathrm{~S}$ & 9 & 10 & 9 & 9.6 \\
\hline & B & 6 & 6 & 9 & 7 & B & 8 & 8 & 9 & 8.3 \\
\hline & $\mathrm{F}$ & 6 & 6 & 8 & 6.6 & $\mathrm{~F}$ & 8 & 8 & 10 & 8.6 \\
\hline \multirow[t]{3}{*}{ Smoothness } & $\mathrm{S}$ & 6 & 6 & 8 & 6.6 & $\mathrm{~S}$ & 8 & 8 & 10 & 8.6 \\
\hline & B & 6 & 6 & 8 & 6.6 & B & 8 & 8 & 9 & 8.3 \\
\hline & $\mathrm{F}$ & 6 & 6 & 8 & 6.6 & $\mathrm{~F}$ & 8 & 8 & 9 & 8.3 \\
\hline \multirow[t]{2}{*}{ Fit } & $\mathrm{S}$ & 6 & 6 & 8 & 6.6 & $\mathrm{~S}$ & 9 & 10 & 9 & 9.3 \\
\hline & $\mathrm{B}$ & 6 & 6 & 8 & 6.6 & B & 8 & 8 & 9 & 8.3 \\
\hline
\end{tabular}

Note: F, Front view; S, Side view; B, Back view

Table 4 Evaluation of Top and Pants

\begin{tabular}{|c|c|c|c|c|c|c|c|c|c|c|}
\hline & \multicolumn{4}{|c|}{ Avatar 1} & \multirow{2}{*}{$\begin{array}{l}\text { Mean } \\
9.3\end{array}$} & \multicolumn{4}{|c|}{ Avatar 2} & \multirow{2}{*}{$\begin{array}{l}\text { Mean } \\
9.6\end{array}$} \\
\hline & $\mathrm{F}$ & 9 & 10 & 9 & & $\mathrm{~F}$ & 9 & 10 & 9 & \\
\hline \multirow[t]{3}{*}{ Appearance } & $\mathrm{S}$ & 9 & 10 & 9 & 9.3 & $\mathrm{~S}$ & 10 & 10 & 9 & 9.6 \\
\hline & $\mathrm{B}$ & 9 & 8 & 9 & 8.6 & $\mathrm{~B}$ & 10 & 10 & 9 & 9.6 \\
\hline & $\mathrm{F}$ & 10 & 10 & 9 & 9.6 & $\mathrm{~F}$ & 10 & 10 & 9 & 9.6 \\
\hline \multirow[t]{3}{*}{ Smoothness } & $\mathrm{S}$ & 10 & 10 & 9 & 9.3 & $\mathrm{~S}$ & 10 & 10 & 9 & 9.6 \\
\hline & B & 9 & 9 & 9 & 9 & B & 10 & 10 & 9 & 9.6 \\
\hline & $\mathrm{F}$ & 10 & 10 & 9 & 9.6 & $\mathrm{~F}$ & 10 & 10 & 9 & 9.6 \\
\hline \multirow[t]{2}{*}{ Fit } & $\mathrm{S}$ & 10 & 10 & 9 & 9.6 & $\mathrm{~S}$ & 10 & 10 & 9 & 9.6 \\
\hline & B & 9 & 8 & 9 & 8.6 & B & 10 & 10 & 9 & 9.6 \\
\hline
\end{tabular}

Note: F, Front view; S, Side view; B, Back view

Table 5 Evaluation of Women's Dress

\begin{tabular}{|c|c|c|c|c|c|c|c|c|c|c|}
\hline & \multicolumn{4}{|c|}{ Avatar 1} & \multirow{2}{*}{$\begin{array}{l}\text { Mean } \\
9.6\end{array}$} & \multicolumn{4}{|c|}{ Avatar 2} & \multirow{2}{*}{$\begin{array}{l}\text { Mean } \\
10\end{array}$} \\
\hline & $\mathrm{F}$ & 10 & 10 & 9 & & $\mathrm{~F}$ & 10 & 10 & 10 & \\
\hline \multirow[t]{3}{*}{ Appearance } & $\mathrm{S}$ & 9 & 9 & 9 & 9 & $\mathrm{~S}$ & 10 & 10 & 10 & 10 \\
\hline & B & 7 & 7 & 10 & 8 & B & 10 & 10 & 10 & 10 \\
\hline & $\mathrm{F}$ & 9 & 10 & 9 & 9.3 & $\mathrm{~F}$ & 10 & 10 & 10 & 10 \\
\hline \multirow[t]{3}{*}{ Smoothness } & $\mathrm{S}$ & 9 & 10 & 9 & 9.3 & $\mathrm{~S}$ & 10 & 10 & 10 & 10 \\
\hline & B & 9 & 10 & 9 & 9.3 & B & 10 & 10 & 10 & 10 \\
\hline & $\mathrm{F}$ & 9 & 10 & 10 & 9.6 & $\mathrm{~F}$ & 10 & 10 & 10 & 10 \\
\hline \multirow[t]{2}{*}{ Fit } & $\mathrm{S}$ & 9 & 10 & 10 & 9.6 & $\mathrm{~S}$ & 9 & 10 & 10 & 9.6 \\
\hline & B & 9 & 10 & 10 & 9.6 & B & 9 & 10 & 10 & 9.6 \\
\hline
\end{tabular}

Note: F, Front view; S, Side view; B, Back view 
Table 6 Evaluation of Sleeveless Straight Dress

\begin{tabular}{|c|c|c|c|c|c|c|c|c|c|c|}
\hline & \multicolumn{4}{|c|}{ Avatar 1} & \multirow{2}{*}{$\begin{array}{l}\text { Mean } \\
9.6\end{array}$} & \multicolumn{4}{|c|}{ Avatar 2} & \multirow{2}{*}{$\begin{array}{l}\text { Mean } \\
10\end{array}$} \\
\hline & $\mathrm{F}$ & 9 & 10 & 10 & & $\mathrm{~F}$ & 10 & 10 & 10 & \\
\hline \multirow[t]{3}{*}{ Appearance } & $\mathrm{S}$ & 9 & 10 & 9 & 9.3 & $\mathrm{~S}$ & 10 & 10 & 10 & 10 \\
\hline & B & 9 & 10 & 10 & 9.6 & B & 10 & 10 & 10 & 10 \\
\hline & $\mathrm{F}$ & 9 & 10 & 9 & 9.3 & $\mathrm{~F}$ & 10 & 10 & 10 & 10 \\
\hline \multirow[t]{3}{*}{ Smoothness } & $\mathrm{S}$ & 9 & 10 & 9 & 9.3 & $\mathrm{~S}$ & 10 & 10 & 10 & 10 \\
\hline & B & 9 & 10 & 9 & 9.3 & B & 10 & 10 & 10 & 10 \\
\hline & $\mathrm{F}$ & 9 & 10 & 10 & 9.6 & $\mathrm{~F}$ & 10 & 10 & 10 & 10 \\
\hline \multirow[t]{2}{*}{ Fit } & $\mathrm{S}$ & 9 & 10 & 10 & 9.6 & $\mathrm{~S}$ & 9 & 10 & 10 & 9.6 \\
\hline & B & 9 & 10 & 10 & 9.6 & B & 9 & 10 & 10 & 9.6 \\
\hline
\end{tabular}

Note: F, Front view; S, Side view; B, Back view

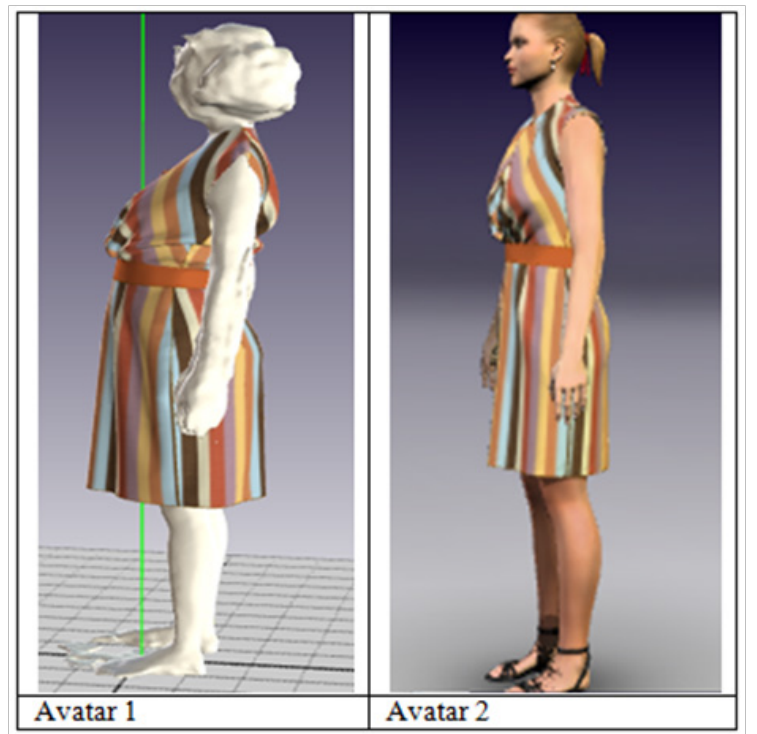

Figure 5 Women's dress simulation on Avatar I (left) and Avatar 2 (right)

\section{Discussion and conclusion}

Findings indicate a low range of differences in appearance, smoothness and fit for the fitting simulations on the two avatars, but overall, the Avatars 2 scored higher (Table 7). Qualitative comments include the garment fabrics on the Avatars 2 were consistently smooth, stiff and close to the body. In contrast, the fabrics on the personalized avatars displayed more realistic fabric creases, drape and relationship of the fabric to the body. Additionally, the images of the Optitex avatar were more aesthetically pleasing. The surface of the personalized avatar and some of the contour areas of the figure need more refinement. Also, the personalized avatar showed more realistic differences in body types and proportions. As affordable, 3D body scanning home systems improve and become available, consumers and designers, can look forward to improved garment fit through virtual applications., ${ }^{9,11,12}$ Personalized avatars could be used to evaluate virtual try on styles, ${ }^{13}$ increase consumer satisfaction and help apparel manufacturers increase sales.

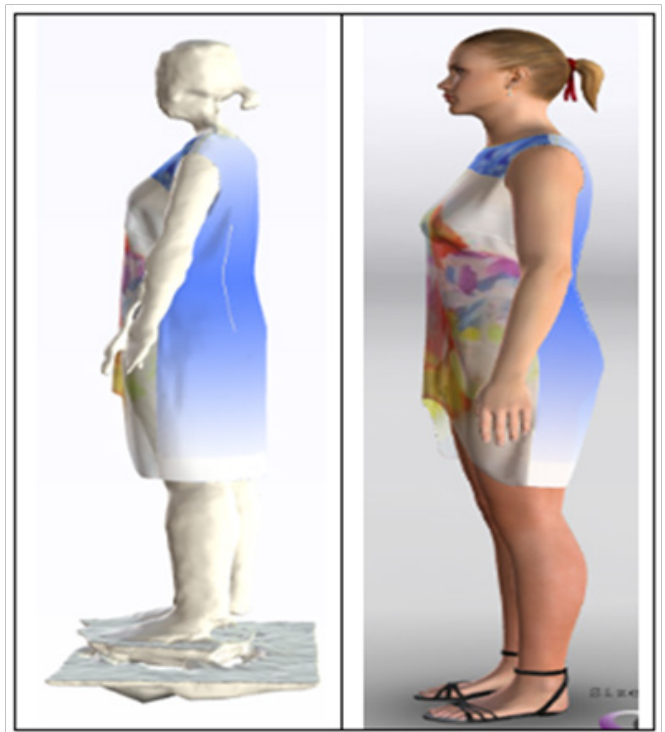

Figure 6 Women's dress simulation on Avatar I (left) and Avatar 2 (right)

Table 7 Grand Means

\begin{tabular}{lllll}
\hline & \multicolumn{2}{l}{ Avatars 1 } & \multicolumn{2}{l}{ Avatars 2 } \\
\hline \multirow{4}{*}{ Appearance } & F & 8.7 & F & 9.6 \\
& S & 8.8 & S & 9.8 \\
& B & 8.3 & B & 9.6 \\
Smoothness & S & 8.5 & S & 9.6 \\
& F & 8.6 & F & 9.6 \\
& B & 8.5 & B & 9.6 \\
& & & & \\
Fit & F & 8.8 & F & 9.4 \\
& S & 8.8 & S & 9.5 \\
& B & 8.7 & B & 9.4 \\
\hline
\end{tabular}


In the first research question, does using a 3D body scanning system such as Kinect to scan oneself and create an avatar versus using a customized programmed avatar in the design process influence a better visualization of fit? It is not 100 percent accurate. But according to the professionals' evaluation, a highly acceptable rate was evident. Referring to the second research question, do different virtual avatars have different fitting results? The results suggest fit is similar in most areas, such as the upper torso. But despite the overall similarity in fit, differences appeared in the drape of the fabric and certain curved areas, such as in the lower torso and at the waist area.

\section{Implications}

There is a lack of empirical examination of fitting simulations on virtual avatars. This exploratory study increases the understanding of the reliability and accuracy of fitting simulations. Further understanding can relate to body type, garment styles and other virtual technologies. Understanding body types can help to create better and normal computerized avatars or use more personalized avatars to make clothing virtual try-on easier. In this study, body scanning and avatar development were conducted on the campus setting by young students of standard sizes. Other age groups and body types may suggest other fitting problems and needs. Additionally, a wider range of styles may affect fitting issues. Furthermore, digitalization of personal avatars will allow personal fitting evaluations to meet personal needs of many different types.

\section{Acknowledgements}

The authors are grateful to the College of Tropical Agriculture and Human Resources for the partial funding of this research.

\section{Conflict of interest}

Authors declare there is no conflict of interest in publishing the article.

\section{References}

1. Istook CL, Hwang S. 3D body scanning systems with application to the apparel industry. $J$ Fashion Marketing \& Management. 2001;5(2):120-132.
2. Jones SG, Schmidt A, Rossi C. 3D scanning systems. J New Technologies. 2010;5(11):35-38.

3. Kim J, Forsythe S. Adoption of virtual try-on technology for online apparel shopping. J Interactive Marketing. 2008;22(2):45-59.

4. Lee Y, Damhorst ML, Lee M, et al. Older women's fit and style concerns and their attitudes toward the use of 3D body scanning. Clothing \& Textiles Research J. 2012;30(2):102-118.

5. Nam J, Hamiln R, Gam HJ, et al. The fashion-conscious behaviours of mature female consumers. International J Consumer Studies. 2006;31(1):102-108.

6. Nantel J. My virtual model: Virtual reality comes into fashion. $J$ Interactive Marketing. 2004;18(3):73-86.

7. Magnenant-Thalmann N, Kevelham B, Volino P, et al. 3D webbased virtual try on of physically simulated clothes. Computer-Aided Design \& Applications. 2011;8(2):163-174.

8. Lin S, Johnson R, Stricker D, et al. Exploratory analysis of college students' satisfaction of body scanning with Kinect. Proceedings of $3^{\text {rd }}$ International Conference on Body Scanning Technologies. Lugano: Switzerland; 2012:302-306.

9. Lin S, Mammel K. An analysis of Digital 3D body imaging technology. Proceedings of the 2nd International Conference on 3D Body Scanning Technologies. Lugano: Switzerland; 2011. p. 58-65.

10. Loker S, Cowie L, Ashdown S, et al. Female consumers' reactions to body scanning. Clothing Textile \& Research J. 2004;22(4):151-160.

11. Loker S, Ashdown S, Cowie L, et al. Consumer interest in commercial application of body scan data. J Textile, Apparel Technology \& Management. 2004;4(1):1-13.

12. Song HK, Ashdown SP. Female apparel consumers' understanding of body size and shape: Relationship among body measurements, fit, satisfaction and body cathexis. Clothing \& Textiles Research $\mathrm{J}$. 2013;31(3):143-156.

13. Suh K, Kim H, Suh EK. What if your avatar looks like you? Dual-congruity perspectives for avatar use. MIS Quarterly. 2011;35(3):711-729. 